\title{
Immune-checkpoint inhibitor anti-PD1 aggravates colitis- associated colorectal cancer without enhancing intestinal inflammation
}

\author{
Maxime Collard $^{1,2}$, Nathalie Guedj ${ }^{3}$, Julien Tourneur-Marsille ${ }^{1}$, Miguel Albuquerque ${ }^{3}$, Léon Maggiori ${ }^{1,2}$, Pascal Hammel $^{4}$, Xavier Treton ${ }^{1,5}$, \\ Yves Panis ${ }^{1,2}$ and Eric Ogier-Denis ${ }^{1 *}$ \\ ${ }^{1}$ Université de Paris, Centre de Recherche sur l'Inflammation, INSERM, France \\ ${ }^{2}$ Department of Colorectal Surgery, Beaujon Hospital, University of Paris, France \\ ${ }^{3}$ Department of Pathology, Beaujon Hospital, University of Paris, France \\ ${ }^{4}$ Department of Digestive and Medical Oncology, Beaujon Hospital, University of Paris, France \\ ${ }^{5}$ Department of Gastroenterology, Beaujon Hospital, University of Paris, France
}

\begin{abstract}
Introduction: Immunity of colitis-associated colorectal cancer (CAC) differs fundamentally from that of the sporadic form. The aim of this study was to evaluate whether this difference could potentiate the efficacy of immune-checkpoint inhibitor anti-PD1 against CAC.

Methods: CAC tumorigenesis was induced by azoxymethane (AOM) followed by three cycles of dextran sodium sulfate (DSS) in mice. Two weeks after the end of DSS, mice were treated with anti-PD1 antibody $(n=9)$ or with isotype antibody $(n=9)$. The severity of clinical and histological colitis, tumor counts, and infiltration of CD8+ T-lymphocytes and neutrophils were compared.

Results: The anti-PD1 antibody did not aggravate the colitis as exemplified by the absence of differences in weight loss ( $\mathrm{p}=0.424)$ and in the standardized pathological scores $(\mathrm{p}=1.000)$ compared to those of the control. On macroscopic examination, the median number of tumors was 24.0 [21.5/31.0] in treated mice and 17.0 [4.0/23.5] in controls $(\mathrm{p}=0.037)$. The percentage of tumor tissue within the entire colonic epithelium was significantly higher in treated mice $(33.1 \%[27.2 / 39.0])$ than in controls $(15.3 \%$ [8.1/25.0]) ( $\mathrm{p}=0.003)$. The intra-tumoral CD8+ T-lymphocyte density was similar between the two groups ( $\mathrm{p}=0.546)$. In contrast, CD8+ T-lymphocyte density was significantly higher in non-tumor colonic epithelium in treated mice than in controls $(\mathrm{p}=0.019)$. Regarding innate immunity, neutrophil density was similar within the tumors $(\mathrm{p}=0.864)$ and augmented in non-tumor colonic epithelium in treated mice compared with controls $(\mathrm{p}=0.012)$.
\end{abstract}

Conclusion: Unexpectedly, checkpoint inhibitor anti-PD1 treatment of CAC stimulates tumor proliferation without flaring-up the colitis.

\section{Introduction}

The adverse effects of immunotherapy (anti-PD1, anti-PDL1 or anti-CTLA4) are dominated by autoimmune complications, such as fatal forms of colitis [1]. The precautionary principle raised concerns about inflammatory flare-ups that may be induced by these treatments in patients with chronic intestinal inflammation. Therefore, these patients have always been excluded from trials on immunotherapy against cancer, including, of course, colorectal cancer [2]. However, data available in the literature of patients with inflammatory bowel diseases (IBD) treated by immunotherapy do not describe a severe flare-up of digestive inflammation in the majority of cases[3-5].

In the sporadic colorectal cancer, the response to immunotherapy is mainly determined by the presence of a mismatch-repair deficiency [2]. But colitis-associated colorectal cancer (CAC) differs in many ways from sporadic colorectal cancer, particularly with regard to immunity [6]. Although only $10 \%$ of CAC presents a mismatch repair-deficiency [7], we hypothesize that the chronic immune activation occurring during IBD could positively impact the efficacy of immunotherapy against CAC. The objective of this study was to investigate the therapeutic effect of anti-PD1 immunotherapy in a mouse model of CAC.

\section{Materials and methods}

\section{Model animal of CAC}

The CAC model selected was the AOM (Azoxymethane)/DSS (Dextran Sodium Sulfate) murine model [8]. Male BALB/c wild-type mice (Charles River) aged five weeks were given a single intraperitoneal administration of AOM (13.4M; Sigma-Aldrich) at a dose of 10 $\mathrm{mg} / \mathrm{kg}$ of body weight. One week after the injection of AOM, mice were treated with three one-week cycles of $1.5 \%$ DSS (Molecular Weight $=36,000-50,000$; MP Biomedicals) in drinking water separated by a one-week cycle between the first and the second cycle of DSS and by a two-week cycle between the second and the third cycle of DSS of

${ }^{*}$ Correspondence to: Eric Ogier-Denis, Laboratory of intestinal inflammation, center of research on inflammation, UMR1149, INSERM, University of Paris, 16 rue Henri Huchard, 75018, France, Tel: +33157277307 , Fax: +3315727746 , E-mail: eric.ogier-denis@inserm.fr

Key words: anti-PD1, colitis-associated colorectal cancer, immunotherapy, inflammatory bowel disease

Received: March 19, 2020; Accepted: April 08, 2020; Published: April 10, 2020 
drinking water without DSS. From the end of the third cycle of DSS, mice were given drinking water without DSS until they were sacrificed. The mice were weighed at least once a week.

\section{Immunotherapy}

The mice were separated into an Anti-PD1-treated group and a Control group. The Anti-PD1-treated group received five intraperitoneal injections of anti-PD1 antibodies (InVivoMAb anti-mouse PD-1, Clone: RMP1-14, BioXCell, $100 \mu \mathrm{g}$ /injection) with a 3.5-day interval between each. The first injection was administered two weeks after the end of the third cycle of DSS. The Control group received five intraperitoneal injections of IgG2a isotype control (InVivoMAb rat, anti-trinitrophenol, BioXCell) according to the same chronology as the anti-PD1 injections. All mice in both groups were sacrificed 1.5 weeks after their last injection by cervical dislocation.

This experiment was approved by the local Animal Ethics Review Committee (nºPAFIS\#14004-2018030914101923v5).

\section{Pathology}

Immediately after sacrifice, the entire colon was removed and opened in a longitudinal direction to quantify the number of tumors visible by macroscopic examination on the entire colonic mucosa. The colon was fixed for $24 \mathrm{~h}$ in $10 \%$ formalin and then embedded in paraffin as "Swiss rolls" containing the full-length organ. Paraffin-embedded sections $(5 \mu \mathrm{m})$ were deparaffinized and stained with $\mathrm{H} \& \mathrm{E}$ reagent. The number of tumors visible on the H\&E-stained slides containing the full-length colon of each mouse were counted. The histological severity of colitis was assessed by a standardized score previously described [9]. These pathological analyses were performed blindly by a trained gastrointestinal pathologist (NG).

In addition, these slides were digitized (Scanscope AT turbo, Leica) and analyzed with Aperio ImageScope software, and the percentage of tumor tissue within the entire colonic epithelium was calculated for each mouse. This quantification method is an automated observerindependent process.

\section{Immunohistochemistry}

Two slides of each colon were prepared for immunohistochemical analysis using antibodies directed against CD8 (ab203035, Abcam, 1/200 dilution) and myeloperoxidase (MPO) (ab9535, Abcam, 1/50 dilution) to identify CD8+ T-lymphocytes and neutrophils, respectively. Immunostained slides of the whole tissue were digitized (Scanscope AT turbo, Leica). Using the Aperio ImageScope software, the tumors were circumscribed to delineate the entire tumor colonic tissue of each slide (layer 1). In addition, all of the colonic epithelium excluding tumor areas were also circumscribed to delineate the non-tumor colonic tissue (layer 2). These surfaces were quantified using the Aperio Membrane algorithm, and the number of cells that fixed the specific antibody was quantified. Thus, the average density per colon of CD8+ T-lymphocytes and neutrophils in the tumor colonic tissue (layer 1) and in the nontumor colonic tissue (layer 2) was calculated.

\section{Statistics}

Quantitative data were expressed as the median and interquartile range (IQR), and values were compared using the Wilcoxon-MannWhitney test. The correlation between two quantitative variables was carried out by using a combined analysis of the scatter plot and the Spearman's rank correlation coefficient. A p-value of $<0.05$ was considered to be statistically significant. All analyses were performed using Prism 8 GraphPad.

\section{Results}

Anti-PD1-treated and Control groups both included 10 mice. One mouse died in each group during the DSS cycles. Of the 18 mice that received intraperitoneal injections (Anti-PD1-treated $\mathrm{n}=9$ and Control $\mathrm{n}=9$ ), none died before the planned date of sacrifice. The severity of the colitis assessed clinically by weight loss and histologically by a standardized score was similar between these two groups (Table 1).

\section{Tumor assessment}

The percentage of tumor tissue within the entire colonic epithelium was increased significantly in the Anti-PD1-treated group $(33.1 \%$ $[27.2 / 39.0])$ compared to that in the Control group (15.3\% [8.1/25.0]), $(p=0.003)$. In the same way, the number of tumors identified after macroscopic examination and the number of tumors counted during microscopic examination of one longitudinal slide for each colon were significantly more in the Anti-PD1-treated group ( $\mathrm{p}=0.037$ and $\mathrm{p}=0.015$ respectively) (Figure 1). The data are presented in table 1.

\section{Immunohistochemistry}

The number of intra-tumoral CD8+ T-lymphocyte density was unaffected by the checkpoint inhibitor treatment compared to that of the Control group ( $\mathrm{p}=0.546$ ). In contrast, CD8+ T-lymphocyte density was significantly higher in the non-tumor colonic epithelium in the AntiPD1-treated group ( $\mathrm{p}=0.019)$. Regarding innate immunity, neutrophil
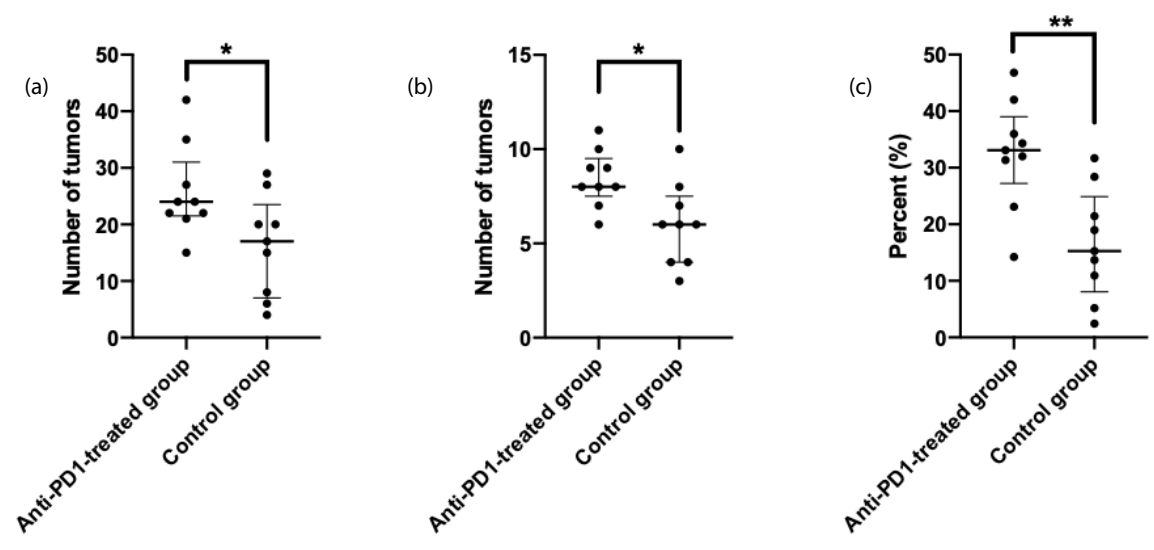

Figure 1. Tumor quantification by macroscopic examination (1-A), by microscopic examination of colon longitudinal slides containing the full-length organ (1-B) and by computerized quantification of the percentage of tumor tissue within the entire colonic epithelium (1-C). 
Table 1. Colitis, tumors and immune infiltration between the Anti-PD1-treated and Control groups

\begin{tabular}{|c|c|c|c|}
\hline & $\begin{array}{l}\text { Anti-PD1-treated group } \\
\qquad \mathrm{n}=9\end{array}$ & $\begin{array}{c}\text { Control group } \\
n=9\end{array}$ & p-value \\
\hline \multicolumn{4}{|l|}{ Colitis } \\
\hline 1st DSS cycle: variation of weight $(\mathrm{g})$ & $+1.0[+0.2 /+2.1]^{1}$ & $+1.3[+1.2 /+2.2]$ & 0.448 \\
\hline 2nd DSS cycle: variation loss of weight (g) & $+0.2[-0.5 /+0.6]$ & $+0.6[0.0 /+1.0]$ & 0.286 \\
\hline 3rd DSS cycle: variation of weight (g) & $-1.1[-2.2 /+0.3]$ & $-0.1[-0.7 /+0.9]$ & 0.424 \\
\hline Treatment period ${ }^{2}$ : variation of weight $(\mathrm{g})$ & $-0.6[3.7 /+1.1]$ & $0[-0.7 /+0.9]$ & 0.424 \\
\hline Histological scoring of colitis & $2.0 / 10[2.0 / 2.5]$ & $2.0 / 10[2.0 / 2.0]$ & 1.000 \\
\hline \multicolumn{4}{|l|}{ Tumors } \\
\hline Macroscopic examination: Number of tumors & $24.0[21.5 / 31.0]$ & $17.0[4.0 / 23.5]$ & 0.037 \\
\hline Microscopic examination of one colon Longitudinal slide: Number of tumors & $8.0[7.5 / 9.5]$ & $6.0[4.0-7.5]$ & 0.015 \\
\hline Percentage of tumor tissue within the entire colonic epithelium (\%) & $33.1[27.2 / 39.0]$ & $15.3[8.1-25.0]$ & 0.003 \\
\hline \multicolumn{4}{|l|}{ Immune infiltration } \\
\hline \multicolumn{4}{|l|}{ Density of CD8+ T-Lymphocyte-8 (cells/mm2) } \\
\hline In the tumor colons & $3.3[2.3 / 5.0]$ & $3.8[2.3 / 6.0]$ & 0.546 \\
\hline In the non-tumor colons & $3.8[2.5 / 9.4]$ & $1.3[0.8 / 3.1]$ & 0.019 \\
\hline \multicolumn{4}{|l|}{ Density of Neutrophils (MPO+) (cells/mm2) } \\
\hline In the tumor colons & $17.9[5.0 / 35.0]$ & $12.1[6.8 / 25.4]$ & 0.864 \\
\hline In the non-tumor colons & $26.1[3.6 / 69.5]$ & $2.5[1.6 / 4.7]$ & 0.012 \\
\hline
\end{tabular}

${ }^{1}$ median [IQR25/IQR75]

${ }^{2}$ Treatment period: from the first injection (anti-PD1 or IgG2a control) until sacrifice

density was similar within the tumors $(\mathrm{p}=0.864)$ and augmented in non-tumor colonic epithelium in treated mice compared with that of the controls $(\mathrm{p}=0.012)$ (Table 1$)$.

After pooling mice from both groups, neutrophil density was positively correlated with CD8+ T-lymphocyte density in non-tumor colonic epithelium (Spearman coefficient $\mathrm{r}=+0.607, \mathrm{p}=0.019$ ). On the scatter plot (Figure 2), mice treated with anti-PD1 were easily distinguished from Control mice by the concomitant increase in CD8+ T-lymphocytes and neutrophil density in non-tumor colonic epithelium.

\section{Discussion}

The results of this study showed that anti-PD1 treatment did not provide any tumor regression in a model of CAC in mouse. On the contrary, this immunotherapy even strengthened tumor proliferation without triggering a clinical or histological flare-up of colitis.

In a previous study by Yassin et al. the administration of anti-PD1 in mice at the same time as the DSS resulted in an exacerbation of colitis [10]. However, the clinical or histological colitis was not aggravated after anti-PD1 treatment in our study, but immune infiltration by both neutrophils and CD8+ T-lymphocytes significantly increased in the non-tumor colonic epithelium. One of the major differences between these two approaches is the injection time of the treatment. While these authors favor the injection of anti-PD1 during the acute phase of DSS treatment, we treated the mice two weeks after the removal of DSS during the chronic phase of colitis. These data in mice are consistent with the cases reported in humans, namely that this treatment does not appear to worsen colitis when administered during the latent phases of the disease in the majority of cases [3-5]. In addition, Yassin et al. [10] did not detect the aggravation of tumor proliferation that we have highlighted, and the difference in the protocol of anti-PD1 injection may account for this difference.

Several hypotheses can explain the absence of tumor regression to anti-PD1 that we observed. First of all, CAC is characterized by low tumor infiltrating lymphocytes even with a mismatch-repair deficiency [11], which is correlated with a non-responder profile to immunotherapy in sporadic colorectal cancer [2]. In other respects, different bacterial

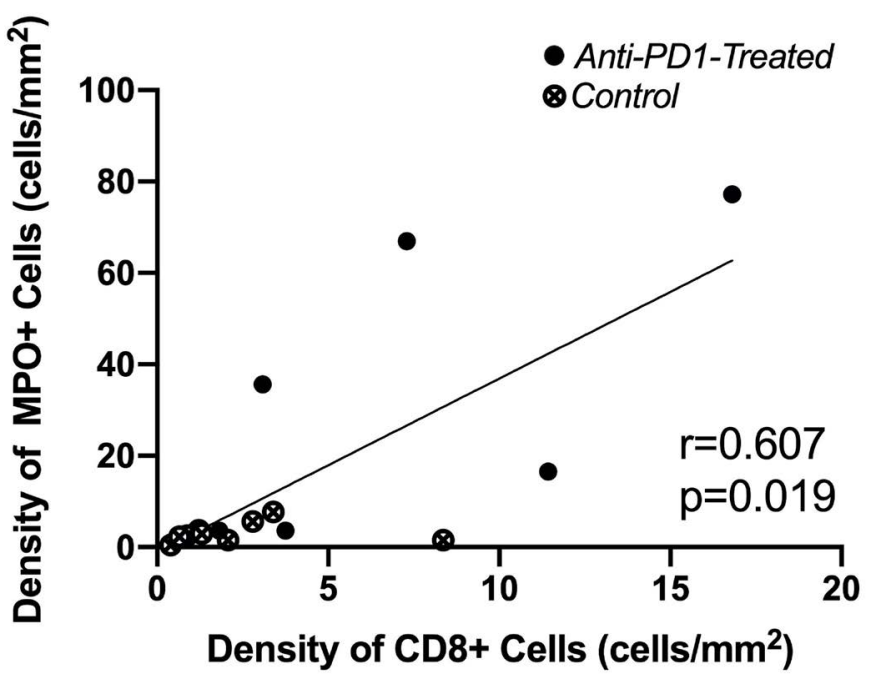

Figure 2. Scatter plot exploring the correlation between CD8+ cell density and MPO+ cell density in non-tumor colonic epithelium.

strains have been identified from healthy donors capable of inducing CD8+ T cell activation in the intestine and enhancing the therapeutic efficacy of immune checkpoint inhibitors [12]. Thus, the loss of efficacy of treatment may be partly related to the presence of intestinal dysbiosis in IBD patients [13].

In our series, an unexpected effect of the checkpoint inhibitor treatment was the worsening of tumor proliferation, and the injection of anti-PD1 did not modify the tumor infiltration by CD8+ T-lymphocytes. Yost et al. have characterized the ability of CD8+ T-lymphocytes to recruit new T-lymphocytes after activation by anti-PD1 [14]. In our model of CAC, this process did not take place in the tumor but did occur in the non-tumor colonic epithelium. In addition, neutrophils were recruited to the non-tumor colonic epithelium and the neutrophil recruitment was proportionally correlated with CD8+ T-lymphocyte recruitment. This activation of innate and adaptive immunity on a quiescent disease is likely to explain the stimulation of proliferation of these tumors that develops during chronic inflammation. 
In conclusion, this pre-clinical study in mice highlights an unexpected effect of checkpoint inhibitors leading to a stimulation of tumor proliferation of CAC. While no worsening of clinical or histological colitis was observed, suppressing the PD1-PDL1 interaction appears to promote the development of CAC.

Competing interests: All the authors declare no potential conflict of interest in relation with this work.

\section{Funding}

This work was supported by grants from the French Institute of Health INSERM, the "Fondation de l'Avenir" AP-RM-17-008, the "Association François Aupetit (AFA)", the "Université de Paris", the "Ligue contre le cancer" and the Investissements d'Avenir programme ANR-11-IDEX-0005-02, Sorbonne Paris Cité, Laboratoire d'excellence INFLAMEX to EOD.

\section{Authors' contributions}

Conception and design of the study: M. Collard, N. Guedj, L. Maggiori, P. Hammel, X. Treton, Y. Panis, E. Ogier-Denis. Acquisition of data: M. Collard, J. Tourneur-Marsille, M. Albuquerque. Analysis and interpretation of data: M. Collard, N. Guedj, E. Ogier-Denis. Drafting the article: M. Collard, N. Guedj, J. Tourneur-Marsille. Revising it critically for important intellectual content: M. Albuquerque, L. Maggiori, P. Hammel, X. Treton, Y. Panis, E. Ogier-Denis. Final approval: All authors.

\section{References}

1. Prieux-Klotz C, Dior M, Damotte D, Dreanic J, Brieau B, et al. (2017) Immune Checkpoint Inhibitor-Induced Colitis: Diagnosis and Management. Target Oncol 12: 301-308. [Crossref]

2. Le DT, Uram JN, Wang H, Bartlett BR, Kemberling H, et al. (2015) PD-1 Blockade in Tumors with Mismatch-Repair Deficiency. N Engl J Med 372: 2509-2520. [Crossref]
3. Johnson DB, Sullivan RJ, Ott PA, Carlino MS, Khushalani NI, et al. (2016) Ipilimumab Therapy in Patients With Advanced Melanoma and Preexisting Autoimmune Disorders. JAMA Oncol 2: 234-240. [Crossref]

4. Menzies AM, Johnson DB, Ramanujam S, Atkinson VG, Wong ANM, et al. (2017) Anti-PD-1 therapy in patients with advanced melanoma and preexisting autoimmune disorders or major toxicity with ipilimumab. Ann Oncol 28: 368-376. [Crossref]

5. Abu-Sbeih H, Faleck DM, Ricciuti B, Mendelsohn RB, Naqash AR, et al. (2020) Immune Checkpoint Inhibitor Therapy in Patients With Preexisting Inflammatory Bowel Disease. J Clin Oncol 38: 576-583. [Crossref]

6. Katoh H, Wang D, Daikoku T, Sun H, Dey SK, et al. (2013) CXCR2-expressing myeloid-derived suppressor cells are essential to promote colitis-associated tumorigenesis. Cancer Cell 24: 631-644. [Crossref]

7. Svrcek M, El-Bchiri J, Chalastanis A, Capel E, Dumont S, et al. (2007) Specific clinical and biological features characterize inflammatory bowel disease associated colorectal cancers showing microsatellite instability. J Clin Oncol 25: 4231-4238. [Crossref]

8. De Robertis M, Massi E, Poeta ML, Carotti S, Morini S, et al. (2011) The AOM/DSS murine model for the study of colon carcinogenesis: From pathways to diagnosis and therapy studies. J Carcinog 10: 9. [Crossref]

9. Johansson ME, Gustafsson JK, Holmen-Larsson J, Jabbar KS, Xia L, et al. (2014) Bacteria penetrate the normally impenetrable inner colon mucus layer in both murine colitis models and patients with ulcerative colitis. Gut 63: 281-291. [Crossref]

10. Yassin M, Sadowska Z, Djurhuus D, Nielsen B, Tougaard P, et al. (2019) Upregulation of PD-1 follows tumour development in the AOM/DSS model of inflammation-induced colorectal cancer in mice. Immunology 158: 35-46. [Crossref]

11. Svrcek M, Fontugne J, Duval A, Flejou JF (2013) Inflammatory bowel diseaseassociated colorectal cancers and microsatellite instability: an original relationship. $\mathrm{Am}$ J Surg Pathol 37: 460-462. [Crossref]

12. Tanoue T, Morita S, Plichta DR, Skelly AN, Suda W, et al. (2019) A defined commensal consortium elicits CD8 T cells and anti-cancer immunity. Nature 565: 600605. [Crossref]

13. Kostic AD, Xavier RJ, Gevers D (2014) The microbiome in inflammatory bowel disease: current status and the future ahead. Gastroenterology 146: 1489-1499. [Crossref]

14. Yost KE, Satpathy AT, Wells DK, Qi Y, Wang C, et al. (2019) Clonal replacement of tumor-specific T cells following PD-1 blockade. Nat Med 25: 1251-1259. [Crossref]

Copyright: (C2020 Collard M. This is an open-access article distributed under the terms of the Creative Commons Attribution License, which permits unrestricted use, distribution, and reproduction in any medium, provided the original author and source are credited. 